\title{
Automotive Crash Insight using AMR Sensor System
}

\author{
Sreevishakh.K.P' ${ }^{1}$, Prof.S.P.Dhanure ${ }^{2}$ \\ ME Scholar, Dept. of Electronics \& Communication Engineering, SITS Narhe, Pune, India ${ }^{1}$ \\ Professor, Dept. of Electronics \& Communication Engineering, SITS Narhe, Pune, India ${ }^{2}$
}

\begin{abstract}
Road traffic accidents (RTAs) have turned out to be India's biggest emerging challenge. Road safety is an issue of global concern on human life and property. In India more than 1,37,000 people were killed in road accidents in 2013 alone, which is greater than the number of people killed in all our wars put together. The main aim of this work is to develop an inexpensive and efficient accident prediction and intimation system based on the concept of inherent magnetic feature of vehicle bodies to provide crash insight to vehicles. Anisotropic magneto-resistive (AMR) sensors which are optimized to work in the Earth's magnetic field range and sonar or ultrasonic sensors are adopted for development of the proposed sensor system. AMR sensors are used to measure the magnetic field disturbances caused by vehicles to Earth's inherent uniform magnetic field and to get presence and relative position of vehicles. AMR sensors are able to work at small inter-vehicular distances down to 0 meter having high refreshment rate and highly inexpensive and compact. Achieving zero road accidents is practically impossible, but with the help of effective accident prediction and notification, we can reduce the severity to the minimal level. Here we integrated GSM and GPS based notification system which will be providing the timely intimation including the crash location to the nearby police station or hospitals which will ensure post-trauma medical care within minimum time. Accident severity and vehicles involved can also be predicted by the proposed system. A brief review on technologies to predict the collision between vehicles and notification systems are also covered in this paper.
\end{abstract}

Keywords: Accident prediction, Notification, Magnetometers, AMR, Intelligent transportation system

\section{INTRODUCTION}

Total no of vehicles on road has experienced a remarkable growth during the last decades leading to increased traffic density and increasing the drivers attention requirements. Immediate effect of this situation is an exponential increase in number of accidents. Nowadays vehicles are integral part of human life; we cannot imagine a day without auto-motives. But there is a drastic increase in the number of traffic accidents in India leading to deaths, fatal injuries, disabilities which impose severe socio-economic costs across the world.

\section{ROAD SAFETY: AN EQUITY ISSUE IN} INDIA

India has a highest number of accident deaths among all countries but road safety has not received the attention as it deserves form the Government and public. Road accidents attract little or no media publicity and consideration because they are became usual in daily life scattered temporarily and spatially. Indian Highways are became the top killers of the country than diseases. In 2013 alone 137572 people died in road accidents, 494893 people got seriously injured. UN Decade of Action for Road Safety 2011-2020 conducted a survey found that emergency medical services (EMS), including ambulances and paramedics are not efficient in most parts of India. $50 \%$ of fatalities and be averted if victims got hospitalized with in the first 1 hour which is be considered as the Golden hour and $80 \%$ of road accident victims in India do not receive any medical services during the golden hours. More than $75 \%$ of respondents and

bystanders are unlikely to assist injured victims because of fear of legal hassles. So it demands the need for an inexpensive accident notification system.

Report of Government of India, Ministry of Road Transport Highways, Transport Research Wing, New Delhi found that $77 \%$ of the accidents are due to driver's fault and points out that fatalities and accidents rates are higher in rural portions than urban areas. More than 22\% of road accidents are caused by Trucks, Tempos and Multi axil vehicles [1].The report demands the need of low cost system in existing vehicles for alerting drivers about high speed and rash driving conditions, crash prediction and intelligent airbag deployment to ensure safety. $36 \%$ of road accidents are due to bad weather conditions. which cause the drivers feel hard to recognize nearby vehicles, speed of vehicles and may leads to severe accidents [2].The present trend of providing safety features like air-bags, antilocking braking system, etc. are limited to luxury segments and is too expensive to be incorporated in heavy motor vehicles and lower end segments.

China has higher population than India with almost similar infrastructures achieved great control on the number of road accidents through Emergency room based injury surveillance systems, emergency medicine training to doctors and nurses and Post-Trauma Medical Care: high efficient grid of medical and paramedical facilities for dealing with road accidents. The same system can also apply here to save hundreds of lives every day on Indian 
roads. A comparative analysis of number of accident deaths in India and China during 2005 to 2013 is shown in figure 2. In 2005 both the countries had almost same number of accident deaths. But through continuous improvement in infrastructures and deployment of advanced Intelligent Transportation System they achieved a sharp reduction in the number of road accident deaths as shown in Fig 2.

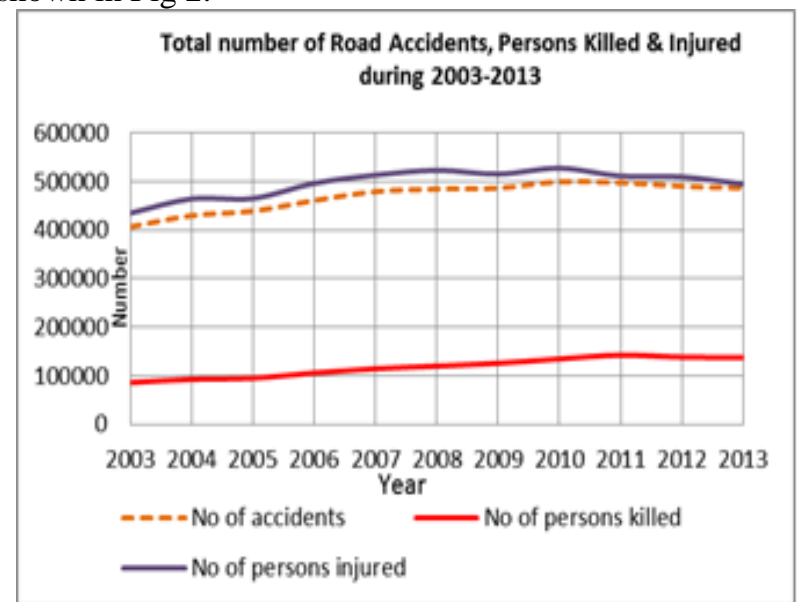

Fig:1 Statistical survey on number of accident deaths in India.

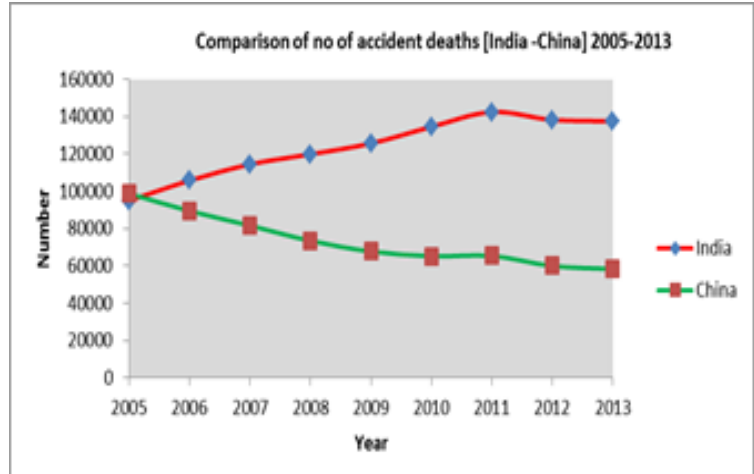

Fig:2 Statistical survey on number of accident deaths in India and China

Intelligent Transportation Systems (ITS) will play significant role in reducing number of fatalities in road accidents. Achieving zero accident is comparatively difficult task, but we can reduce the time between accident and rescue operation usually called as Golden hour. Advanced Automated Crash Notification (AACN) systems use telemetric data from vehicles to notify emergency services of a collision. Now in 21st century leading automobile manufactures introduced Adaptive cruise control and Advanced crash warning and collision avoidance systems in their high end models only. ACC systems mainly based on radar sensor fusion keep safe driving distance from vehicle ahead by adjusting the speed of the vehicle. It doesn't depend on satellite, roadside infrastructures, cooperative support from other vehicles like V2V [Vehicle to Vehicle communication system].

Speed is identified as the key risk factor influencing both chances of road accidents and severity of injuries resulted from crashes. In developed countries $30 \%$ of road accidents are due to speed of vehicles, the same contributes $50 \%$ of road accidents in developing countries like India. As the speed increases the response time for drivers reduced to fraction of seconds. Total breaking response time combine how long it takes the driver to identify a hazard requiring immediate response, time taken to move foot from accelerator to brake pedal and vehicle braking capacity shown in figure 3 . Reaction depends on age of drivers, affected by tiredness, alcohol etc.

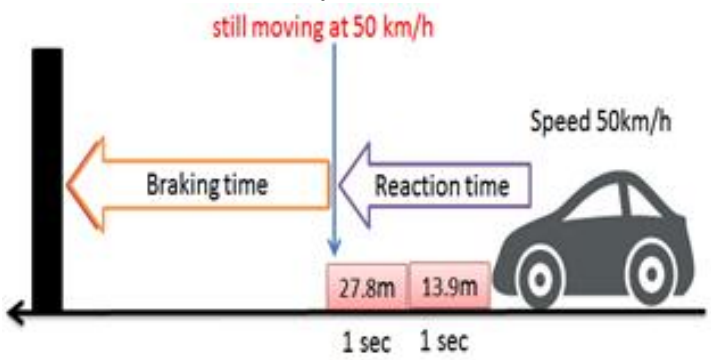

Fig:3 Braking time effect.

The collision prediction system module functionality is to predict the possibility of accident by considering the relative velocity of vehicles. The speed and relative positioning of the vehicles are the main traffic parameters under consideration. Vision based traffic alarm system for drivers assistance is also considered in the early days and the main intention was to give an alert message to driver predicting the possibility of a harmful traffic situation. In this system, object tracking and recognition are the main milestones. Stereo-analysis and classification stages are used in the olden days for implementing the vision based traffic warning system [3]. In the later stages, an advanced scheme for object detection by combining the stereo analysis and motion analysis also proposed. Later, by exploring the features of radar and laser sensor modules, the crash prediction system improved a lot in terms of reliability and functionality. The latest development in the collision detection module is the introduction of AMR sensor by exploiting the properties of magnetic field. We will discuss the different schemes associated with the crash prediction module in details in the later part of this paper.

\section{OVERVIEW ON THE EXISTING CRASH PREDICTION AND NOTIFICATION SYSTEM.}

Even though the history of safety implementation in vehicles started early in the years of 1930, The consideration of real time model development using all possibilities of automation started in the early years of 1990 's. The main concentration was to improve the high way capacity and safety with automation in highway and vehicle level [4]. The advanced development happened in the electronics and sensor technologies like Radar and MEMS [Micro Electro Mechanical System] added advantages to the research in vehicle automation area. Mitsubishi Motors introduced Lidar based distance warning system for trucks and buses, the objective of which is to predict a possibility of a rear-end collision and urge the driver to be careful in 1992. Adaptive cruise 
control and Advanced crash warning and collision avoidance systems are now available in almost all leading vehicles but in their high end model only. Some of them are BMW with Active cruise control[ACC] with stop and go functions. Audi introduced Full speed range ACC plus based on radar and World first GPS-guided radar ACC. DISTRONIC PLUS with Steering Assist-Traffic jam assist is integrated in Mercedes selected models only.

Current Adaptive cruise control and advanced crash warning and collision avoidance systems are mainly based on Radar and Laser sensors. Following are the famous driver assistance system existing around the world: e-Call is a European initiative intended to bring rapid assistance to motorists involved in accidents anywhere in the European Union. It will send airbag deployment status, impact sensor values and GPS coordinates to local emergency services. On-Star introduced by General Motors that provides subscription-based Communication system in-vehicle security, hands free calling. Ford SYNC and Lexus Link also provide telematics services to users. The paper [5] proposes a novel fuzzy based accident prevention system. The distance and relative speed of the vehicle in front is measured by the help of radar sensors. The simulated relative distance and relative speed measured by the radar sensor are sent into the ANFIS ANFIS [Adaptive Neuro Fuzzy Inference System] controller to simulate the car following performance.

Another Fuzzy based accident prediction system model is proposed by Jeich in the paper [6] in 2005. The previous paper by Jeich considers only the car following vehicles. In the advanced method, he concentrated on the development of car following and lane changing accident model in which the relative speed measured from spread spectrum radar is applied to the controller to predict accident possibility. Fuzzy-based accident prediction system by Venkatesh $\mathrm{K}$ is a module which is utilizing the concept of intelligent learning capabilities of neural network and laser sensors for capturing the data. The data from sensor will be passed to a two-stage prediction systems which will first prioritize and then analyses the possibility of accident prediction. The system is intended to give a warning alert to the driver once the possibility of accident is confirmed [7].

A vehicle Frontal Collision Warning System (FCWS) based on improved target tracking and threat assessment for accident prediction utilizing radar and Lidar sensors are described in the paper [8]. Radar and Lidar sensors are used to capture the longitudinal and lateral information of the objects from the vehicle. In this model, when a target is detected by both sensors, the fused longitudinal position is a weighed sum of both radar and Lidar, and the lateral position is from the Lidar The combination of Radar and Lidar helps in getting better target positions and to provide robustness under severe weather. Based on the information obtained from the sensors, if the potential collision is detected, warning signal will be passed to the driver for his immediate attention. Another important development in the accident detection model which can detect the presence of animal on road during night travel. This model [9] uses the features of thermal imaging technology for the object detection. The prototype consists of an infrared thermal temperature image grabbing and processing system, which includes an infrared thermal camera, a frame grabber, an image processing system and a motion tracking system, which includes two motors with their motion control system. By analysing the infrared thermal images which are independent of visible light, the presence of an animal can be determined in either night or day time through pattern recognition and matching.

Airbag deployment is one of the important safety measures embedded in almost all the vehicles. But uncertain deployment of airbags can even cause severe injuries to passengers. This paper [10] proposes effective pre-crash prediction models which ensure that activation of airbags in time. Here radar sensors are used to gather information regarding the object in front to the vehicle and vehicle dynamic control sensors to get details about the vehicles. The possibility of crash is predicted by combining of ACC and VDC sensor information. The time-to-crash information allows the activation of reversible restraint systems like seat belts. The heading angle and the lateral position at the crash moment allow discriminating crash types such as frontal crash, offset crash and oblique crash. The main of this study is to discriminate the possible crash and provide the crash type information to the airbag deployment algorithm before the crash is occurred in advance. This work is able to enhance the performance of the airbag deployment algorithm that the airbags are deployed along the discriminated the crash type.

The work by T.U.AnandSanthosh Kumar1, J. Mrudula [2] proposed an accident prediction which can afford bad weather conditions as well. As they mentioned in the paper, majority of the accidents happens in bad weather conditions. And most of the existing model won't work efficiently in bad weather. IR and ultraviolet sensors are used for the implementation of the accident prediction model. To collect information of weather, temperature sensor and humidity sensors are used. If the humidity level is above the threshold vale set in the model, warning for heavy rain passed to the driver. Similar way, temperature sensor sense the weather and warn the driver for heavy snow conditions. Except the backside, around the vehicles IR and ultraviolet sensors are placed which gather information related to the nearby vehicle. These sensors regularly scan the road for obstacles, if it locate any obstacle in the predefined range, warning alert generated. In their paper [11],Mbachu and Onuora explain their research on the development of a low cost highly reliable accident detection system based on Ultrasonic Sensors. Here in order to measure the distance between the cars and the obstacle, two different methods are used. Time Of Arrival (TOA) method and Angle Of Arrival (AOA) method. In the first method, TOA, the transmitter and receiver located in the vehicle are used for calculating the 
time taken for the propagation of ultraviolet signal from the transmitter to reflect back to the receiver after hitting on the obstacle which can be another vehicle or an object

All the system based on radar or laser sensors have the drawback that the system won't work properly with the bad weather condition such as rain, snow or fog[12].Laser radar(Lidar) have good angular resolution and range. Paper [13] provided influence of different weather conditions on the performance of these sensors. Radar and laser sensors are expensive well over $\$ 1000$. Hence, it is also unaffordable that a number of radar and laser sensors be distributed all around the vehicle in order to predict all the possible types of collisions that can occur.

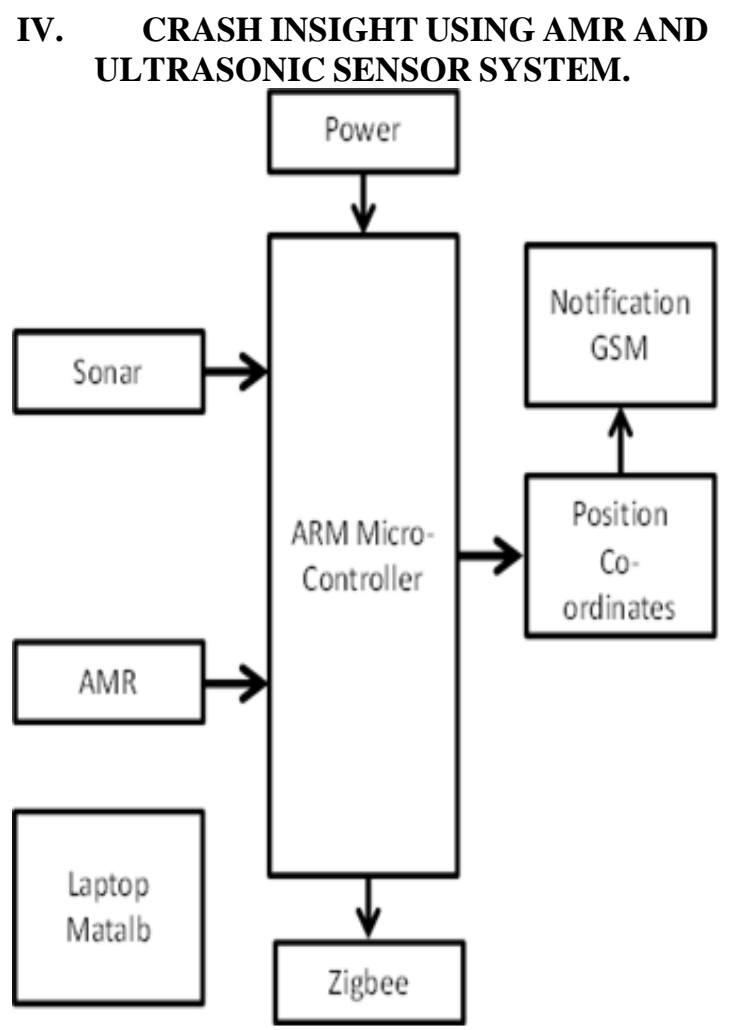

Fig:4 Proposed system block diagram

The main idea of the new proposed system is detect the changes cause to Earth's inherent uniform magnetic field due to vehicle bodies. A vehicle is made of many metallic parts (for example, chassis, engine, body, etc.), which have a residual magnetic field and/or get magnetized in the Earth's magnetic field [15]. AMR sensors are now considered as the best candidate for vehicle detection system as comparatively inexpensive an ease of implementation. Using this technology the power consumption will be less and the system will be able to capture the data within a short region as well. Honeywell triaxial HMC5883L is used in the proposed system development. It is a fully digital AMR sensor with built-in multiplexed ADC. The sensor has 12 bits ADC coupled with low noise AMR sensors and achieves 5 milli-gauss resolution in \pm 8 Gauss Fields. AMR sensors provide a good means of measuring both linear and angular position and displacement in the earth's magnetic field.

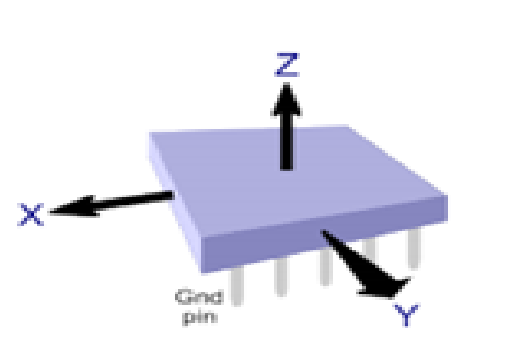

Fig:5 Magnetic fiield orientation of HMC58831

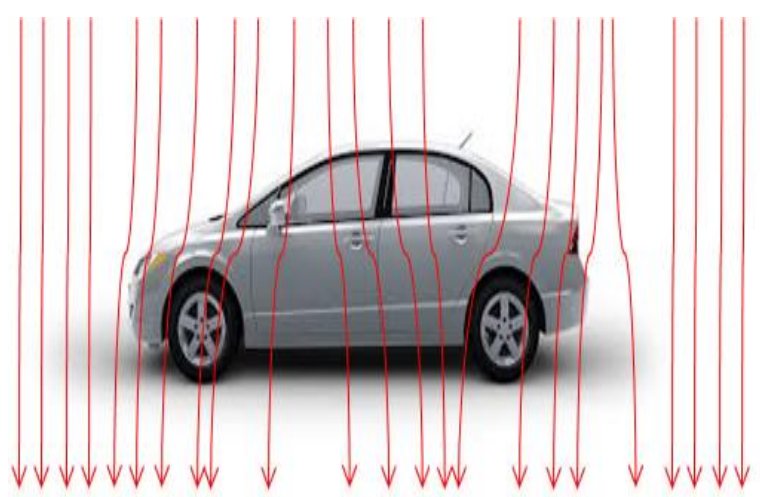

Fig:6 Disturbance caused by vehicle to Earth's uniform magnetic field.

AMR sensors are made by Perm alloy thin films deposited on a silicon substrate in various resistor bridge configurations provide highly predictable outputs when subjected to magnetic fields.Advantages of AMR sensors are low cost, high sensitivity, small size, noise immunity, and reliability over mechanical or other electrical alternatives. The 3-Axis Compass module can measures magnetic fields in three directions or axes, labelled $\mathrm{X}, \mathrm{Y}$, and $\mathrm{Z}$. In its most simple form, it can be used as a basic compass to find earth's magnetic north. The compass module can also sense the relative strength of a nearby magnetic source, such as those caused by magnets or electric field or variation caused by ferromagnetic materials contained in vehicle bodies. As the sensor detects magnetism in three dimensions, we can use it to determine relative distance and direction to these sources. A sonar sensor will directly measure position with respect to itself independent of relative speed. It can measure larger distances compared with the magnetic sensors of several feet but its performance degrade at very short distances below $0.25 \mathrm{~m}$. Also it has a narrow field of view at short distances. A sonar sensor typically has low refresh rate this refreshment rate is adequate for assistance during parking but it is highly inadequate for a crash detection and active passenger protection system. AMR sensors can achieve a very high Bandwidth of $5 \mathrm{MHz}$.

A sensor fusion system is adopted to exploit the advantages of both types of sensors and to overcome their individual drawbacks. In initial state system will use the sonar sensor to update position, since the magnetic sensors are not yet affected by the approaching vehicle. Relative speed of the vehicle can also be calculated during this stage. As soon as the magnetic sensors respond to the approaching vehicle, means vehicle approaching less than 
1 meter inter-vehicular distance region and if the relative speed is higher than the threshold speed system will sense inevitable crash and will send accident position through GPS GSM modules. GPS (Global Positioning System) module outputs will be NMEA (National Marine Electronics Association) format. GGA is used in this model which gives Time, position and fixes related data for a GPS receiver. Standard output format is as follows: \$GPGGA, hhrnmss.sss, dd mm. mmmm, a, dddmm. mmmm, a, x, xx, x.x, x.x, M,,,,xxxx*hh. SIM 300 GSM module isused to send accident location and time of accident to Post trauma medical care unit. It comes with standard RS 232 interface enabling easy communication with Microcontroller and PC.

\section{File Edit View Call Transfer Help

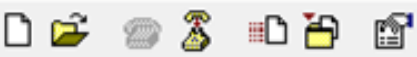

\begin{tabular}{|c|}
\hline $\begin{array}{l}\text { Heading: } 32 \\
\text { Distance: } 175.13 \mathrm{CM} \\
\text { Speed:0.01 Mtrs/Sec } \\
\text { Response Time:5.88 }\end{array}$ \\
\hline $\begin{array}{l}\text { X: } 46.00 \text { Y: } 16.36 \text { 2: } 24.90 \\
\text { Heading: } 32 \\
\text { Distance: } 175.1 \mathrm{CM} \\
\text { Speed:0.16 Mtrs/Sec } \\
\text { Response Time: } 163.71\end{array}$ \\
\hline $\begin{array}{l}\text { X: } 45.55 \text { Y: } 17.00 \text { Z: } 24.69 \\
\text { Heading: } 33 \\
\text { Distance: } 195.41 \mathrm{CM} \\
\text { Speed:0.04 Mtrs } / \mathrm{Sec} \\
\text { Response Time: } 36.51\end{array}$ \\
\hline
\end{tabular}

Fig:7 AMR and sonar reading on Hyper terminal

Experiment is conducted on Wagon $\mathrm{R}$ with proposed system .AMR sensor provided good detection below 0.8 meters as show in Fig 8 since crash prediction at long distance is not reliable. The sensor system can provide 360 degree.

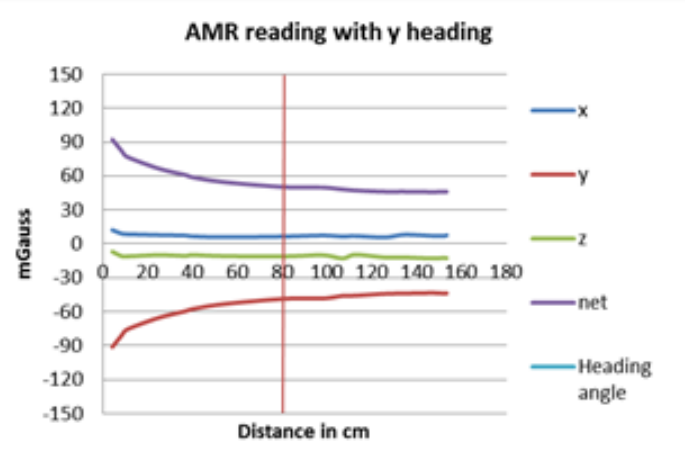

Fig:8 AMR 3 axis reading with Wagon $\mathrm{R}$.



Fig:9 AMR 3 axis reading with Wagon $\mathrm{R}$ from different positions

Fig 9 shows the AMR reading at $5 \mathrm{~cm}$ distance from different parts of the vehicle.

\section{CONCLUSION AND FUTURE SCOPE}

In this paper, we have gone through the various technologies used for automatic accident prediction as well as notification system. For accident prediction system, the excellent features of AMR and sonar sensor lead to the development of a vigilant module. Radar and laser sensors need to be replaced because of their high cost and will not work at short distances. Magneto-resistive sensors are previously used for traffic intensity measurements, vehicle detection in parking applications they are used for the first time in combination with sonar sensors for automotive crash prediction and notification system. We can also predict the intensity of accident using value of AMR sonar sensor fusion. If we can reconstruct accident profile it will be a great advantage. The proposed model is an inexpensive, highly reliable model for accident prediction and alert system.

\section{REFERENCES}

[1]. National Crime Records Bureau (Ministry of Home Affairs) Government of India. June 2014.

[2]. Advanced Accident Avoidance System for Automobiles. T.U.Anand , Santhosh Kumar, J. Mrudula. International Journal of Computer Trends and Technology (IJCTT) - volume 6 number 2Dec 2013.

[3]. Fast Obstacle Detection for Urban Traffic Situations, U. Franke and S. Heinrich IEEE Transactions on intelligent transportation systems, vol. 3, no. 3, September 2002.

[4]. Review of National Automated Highway Research Program, 1998.

[5]. An ANFIS Controller for the Car-Following Collision Prevention System, Jeich Mar, Member, IEEE, and Feng-Jie Lin. IEEE transactions on vehicular technology, vol. 50, no. 4, July 2001

[6]. The Car-Following and Lane-Changing Collision Prevention System Based on the Cascaded Fuzzy Inference System, Jeich Mar, Senior Member, IEEE, and Hung-Ta Lin, IEEE transactions on vehicular technology, vol. 54, no. 3, May 2005.

[7]. NEFCOP: A Neuro-Fuzzy Vehicle Collision Prediction System; Venkatesh, K. ; Dept. of Comput. Sci. Eng., Sri Venkateswara Coll. of Eng., Sriperumbudur . Computational Intelligence for Modelling, Control and Automation, 2005 and International Conference on Intelligent Agents, Web Technologies and Internet Commerce,International Conference on (Volume:1 ); 28-30 Nov. 2005.

[8]. Vehicle Frontal Collision Warning System based on Improved Target Tracking and Threat Assessment.Yimin Wei, Huadong Meng, Hao Zhang, and Xiqin Wang; Proceedings of the 2007 IEEE. 
[9]. Intelligent Transportation Systems Conference, Seattle, WA, USA, Sept. 30 - Oct. 3, 2007.

[10]. Tracking-Based Deer Vehicle Collision Detection Using Thermal Imaging, Debao Zhou, IEEE Member, Matt Dillon and Eil Kwon, Proceedings of the 2009 IEEE International Conference on Robotics and Biomimetics, December 19 -23, 2009, Guilin, China.

[11]. A Pre-crash Discrimination System for an Airbag Deployment Algorithm; Kwanghyun Cho, Seibum B. Choi, Kyungjae Shin and Yousik Yun; 2010 American Control Conference Marriott Waterfront, Baltimore, MD, USA June 30-July 02, 2010.

[12]. A Vehicular Accident Detection and Avoidance system for protecting passengers and vehicles; Mbachu, C. B. \& Onuora, O. N. Department of Electrical and Electronic Engineering, Anambra State University Anambra State, Uli NIGERIA, European Journal of Engineering and Technology Vol. 2 No. 2, 2014 ISSN 20565860 .

[13]. Comparison of Lidar-Based and Radar-Based Adaptive Cruise Control Systems Glenn R. Widmann, Michele K. Daniels, Lisa Hamilton, Lawrence Humm, Bryan Riley, Jan K. Schiffmann, David E. Schnelker and William H. Wishon Delphi Automotive Systems SAE 2000 World Congress Detroit, Michigan March.

[14]. Influences of weather phenomena on automotive laser radar systems,R. H. Rasshofer, M. Spies, and H.Spies,Adv. Radio Sci 9, 4960, 2011 www.advradio-sci.net/9/49/2011/ doi:10.5194/ars-9-492011

[15]. Vehicle Detection and Compass Applications using AMR Magnetic Sensors, Michael J. Caruso, Lucky S. Withanawasam Honeywell, SSEC, 12001 State Highway 55, Plymouth, MN USA 55441.

[16]. The Development of Vehicle Position Estimation Algorithms Based on the use of AMR Sensors, Saber Taghvaeeyan and Rajesh Rajamani. IEEE Transactions on intelligent transportation systems, vol. 13, no. 4, december 2012 